\title{
Evidence for a Young Stellar Population in NGC 5018
}

\author{
Andrew J. Leonardi ${ }^{1}$ \\ CB \#3255, Department of Physics \& Astronomy, University of North Carolina, Chapel Hill, NC \\ 27599-3255 \\ and \\ Guy Worthey \\ Department of Physics \& Astronomy, St. Ambrose University, 518 W. Locust St., Davenport, IA \\ 52803-2829
}

\begin{abstract}
Two absorption line indices, Ca II and $\mathrm{H} \delta / \lambda 4045$, measured from high-resolution spectra are used with evolutionary synthesis models to verify the presence of a young stellar population in NGC 5018. The derived age of this population is $\sim 2.8$ Gyr with a metallicity roughly solar and it completely dominates the integrated light of the galaxy near $4000 \AA$.
\end{abstract}

Subject headings: line: profiles — galaxies: abundances — galaxies: elliptical and lenticular, cD - galaxies: individual (NGC 5018) — galaxies: starburst — galaxies: stellar content

\section{Introduction}

Morphological peculiarities in the optical images of galaxies are now almost invariably taken to be signs of a past tidal interaction or merger. Though theoretical models have had success reproducing tidal tails, shells, and other structures (e.g., Toomre \& Toomre 1972; Quinn 1984; Hernquist \& Quinn 1988; Mihos, Bothun, \& Richstone 1993), in the absence of explicit details of the interaction such as the Hubble types of the progenitors, relative progenitor sizes, and impact parameter, a unique solution is difficult to come by.

Dynamical friction during a collision almost certainly leads to a merger of the stellar systems (Schweizer 1983) and simulations have shown that any accompanying gas will rapidly dissipate to the center during minor mergers involving either ellipticals (Weil \& Hernquist 1993) or disk

\footnotetext{
${ }^{1}$ Visiting Astronomer, Cerro Tololo Inter-American Observatory. CTIO is operated by AURA, Inc. under contract to the National Science Foundation.
} 
galaxies (Mihos \& Hernquist 1994a). If the conditions are right, it is reasonable to expect that this gas inflow will result in star formation. The duration, intensity, and even the starting time of the star formation, however, depend on the morphology of the progenitor and the details of the interaction (Mihos \& Hernquist 1994b). As much post-merger information as possible is needed to help constrain the merger possibilities, including analysis of the resulting young stellar population (YSP).

Unfortunately, unless the merger remnant is presently forming stars at a reasonably vigorous rate (hence producing readily apparent emission lines) or has experienced a very recent and relatively strong episode of star formation placing it in the starburst regime, a YSP can be difficult to detect. Broadband colors and other YSP indicators return to pre-star formation levels very quickly (Bica, Alloin, \& Schmidt 1990; Charlot \& Silk 1994) and many indicators that imply the presence of a YSP can also be explained by intrinsic metallicity differences in the final population of the merger remnants (Bertola, Burstein, \& Buson 1993; hereafter BBB). Ambiguity about whether a YSP even exists or not complicates the details of the merger considerably.

In this paper, we use spectral indices in conjunction with evolutionary synthesis models to detect and determine the age and metallicity of a YSP in the particular case of a possible merger remnant, NGC 5018. It represents an update to the age-dating technique introduced in Leonardi \& Rose (1996; hereafter LR). NGC 5018 is appropriate to the present discussion because there is ongoing uncertainty concerning the presence of a YSP. Certain observations imply the existence of a YSP while others seem to be inconsistent with the presence of a YSP. NGC 5018 and the roots of the controversy are described in $\S 2$. In $\S 3$, a review of the age-dating technique and its refinements are given. The results of the technique applied to NGC 5018 are presented in $\S 4$ and $\S 5$ contains the conclusions.

\section{2. $\quad$ NGC 5018}

NGC 5018 is a member of the Malin \& Carter (1983) catalog of shell elliptical galaxies and is considered a probable merger remnant (Fort et al. 1986). As noted by Schweizer et al. (1990) and BBB, NGC 5018 has an abnormally weak $\mathrm{Mg}_{2}$ index for its luminosity: Its measured $\mathrm{Mg}_{2}$ is 0.209 (Trager et al. 1998) even though the mean $\mathrm{Mg}_{2}-\sigma$ relation suggests $\mathrm{Mg}_{2}=0.301$ for an elliptical galaxy with NGC 5018's measured velocity dispersion of $\sigma=223 \mathrm{~km} \mathrm{~s}^{-1}$ (Bender, Burstein, \& Faber 1993), about 6 standard deviations away from the mean. Although deviations from the line-strength-luminosity relation correlate well with the amount of morphological disturbance in elliptical galaxies (Schweizer et al. 1990), NGC 5018 has abnormally weak line strengths even when this correlation is accounted for. For a class of objects, these authors ruled out metallicity variations in the galaxies as the cause for the correlation due to the physical implausibility of stronger mergers leading to more metal-poor stellar populations in the remnants. Schweizer et al. concluded that mergers produce a YSP which is observed in the decreased line strengths. On a galaxy-by-galaxy basis, however, an intrinsic metallicity variation cannot be ruled out by a low $\mathrm{Mg}_{2}$ 
index alone. In NGC 5018, a low $\mathrm{Mg}_{2}$ index coupled with the lack of an upturn in its UV spectral energy distribution (SED), led BBB to conclude that NGC 5018 consisted of a metal-poor old stellar population, in stark contrast to other ellipticals of the same luminosity. BBB were unable to match both the UV SED observations and the $\mathrm{Mg}_{2}$ index with composite populations created by mixing spectral templates of metal-rich elliptical galaxies and a contaminating YSP template. Only templates containing metal-poor populations approached both observations.

Indirect observational evidence that a YSP does in fact exist in NGC 5018 is extensive. The shells present in its optical image are photometrically bluer than the surrounding parts of the galaxy (Fort et al. 1986) suggesting a younger age for the shells. The detection of an HI gas bridge connecting NGC 5018 with the nearby spiral NGC 5022 (Kim et al. 1988) is evidence of an ongoing interaction while a possible past interaction is implied by a stellar bridge connecting the two and the embedded dust lane in NGC 5018 (Malin \& Hadley 1997). Possible young globular cluster candidates, formed during a past interaction and perhaps only several hundred Myr old, have been observed (Hilker \& Kissler-Patig 1996). Furthermore, Goudfrooij et al. (1994) measured extended $\mathrm{H} \alpha+[\mathrm{N} \mathrm{II}]$ emission in the central region coinciding with the embedded dust lane which they associated with star forming regions. Also, IR emission has been detected in the same area (Jura et al. 1987). Thronson \& Bally (1987) showed in an IR two-color diagram that NGC 5018 lies in a region quite different from that occupied by infrared "cirrus", which is emission from diffuse dust in the interstellar medium of a galaxy. Instead, it is closer to the region where the IR emission from warmer dust associated with HII regions dominates (Helou 1986; Bushouse, Lamb, \& Werner 1988), suggesting a YSP source for the IR emission.

While the observations are compelling, they are not conclusive. The direct detection of the YSP is needed to resolve the issue. BBB chose to observe in the far-UV for exactly that reason, since in principle, this region of the spectrum is dominated by young stars (O'Connell 1988) and the low UV flux level in NGC 5018 led them to the metal-poor scenario. They discounted dust obscuration as the cause because the best available photometry at that time (Fort et al. 1986) showed that the dust lane in NGC 5018 does not extend into the region where their IUE spectrum was taken. Subsequent observations, however (Carollo \& Danziger 1994; Goudfrooij et al. 1994), indicate that not only is dust present throughout the central region but also is patchy in nature, making the reddening effects difficult to ascertain. Carollo \& Danziger (1994) showed that reasonable expectations of dust obscuration and a YSP can explain both the low $\mathrm{Mg}_{2}$ index and the UV flux depletion in the central region of NGC 5018. Both sets of authors concluded that a YSP was a more probable explanation for the observations.

The conflict is illustrated in Figure 1 where we have plotted data from the Lick group (Trager et al. 1998) for NGC 5018 and other systems. The top panel shows a $\lambda 2750-V$ color plotted against the $\mathrm{C}_{2} 4668$ Lick index. The $\mathrm{C}_{2} 4668$ index is more metal-sensitive than $\mathrm{Mg}_{2}$ and is less subject, but not immune, to abundance ratio effects. BBB remarked that NGC 5018's $\mathrm{Mg}_{2}$ index and UV spectrum resembles M32's, even though M32 is a much less luminous galaxy, which led them to surmise a system with an abundance like that of a dwarf galaxy rather than a giant elliptical. The 
UV data here supports this view. As can be seen in the top panel of Figure 1, NGC 5018 has approximately the same UV color but a slightly weaker $\mathrm{C}_{2} \lambda 4668$ index than M32 indicating that NGC 5018 is about twice as old and more metal-poor than M32. The bottom panel of Figure 1 plots $\mathrm{H} \beta$ (uncorrected for any emission fill-in) against $\mathrm{C}_{2} 4668$ and shows NGC 5018 nearly on the same model grid line as M32 implying similar age, but still $\sim 0.15$ dex more metal-poor than M32. Emission corrections for $\mathrm{H} \beta$ would push NGC 5018 vertically upward to younger ages and higher metallicity, worsening the discrepancy in age between the two panels. On the other hand, a correction for UV extinction in the upper panel would make the two panels agree better.

To help resolve the still uncertain nature of NGC 5018, we utilize spectroscopic observations, along with an updated age-dating method to unambiguously show that a YSP is present in NGC 5018. The modeling technique is discussed in $\S 3$. The long-slit spectra of NGC 5018 were acquired at the KPNO 4m telescope in June 1995 by Lewis Jones and kindly provided to us. Four 30-minute exposures were acquired with the R-C spectrograph with grating KPC-22B at second order and the T2KB 2048x2048 CCD. The slit width was 2 arcsec. A 14.5 pixel aperture was extracted from the raw spectrum and with a CCD spatial scale of $\sim 0.69 \operatorname{arcsec} /$ pixel, the aperture size on NGC 5018 is 2 " $x 10$ ". The dispersion of the spectra is $0.7 \AA /$ pxl and the resolution is FWHM $\sim 1.8$ A. Data reductions were done in IRAF. For details see Jones (1999). A representative spectrum, emphasizing the wavelength region of interest, is shown in Figure 2. It has been normalized to unity at $4040 \AA$.

\section{The Age-Dating Technique}

The age-dating technique as described in LR uses two spectral indices developed in Rose $(1984,1985)$. Each index is defined by taking the ratio of counts in the bottoms of two neighboring absorption lines without reference to the continuum levels. The specific absorption lines used are identified on the NGC 5018 spectrum in Figure 2. The first index, $\mathrm{H} \delta / \lambda 4045$, measures the integrated spectral type of a galactic stellar population and is produced from the ratio of the central intensity in $\mathrm{H} \delta$ relative to the central intensity in the neighboring Fe I $\lambda 4045$ line. Note that the way the index is defined, $\mathrm{H} \delta / \lambda 4045$ decreases as $\mathrm{H} \delta$ gets stronger. The second index, Ca II, formed from the ratio of the central intensity of $\mathrm{Ca}$ II $\mathrm{H}+\mathrm{H} \epsilon$ relative to $\mathrm{Ca}$ II $\mathrm{K}$, is constant in stars with a spectral type later than F2 but then decreases dramatically for earlier type stars, reaching a minimum at spectral type A0. It provides an unambiguous signature for stars hotter than F2 in the integrated light of a stellar population.

The indices are computed for single-age theoretical populations from evolutionary synthesis models. When plotted together in the two-dimensional index space, the indices resolve the wellknown degeneracy between a YSP's age and light contribution to a composite stellar population (e.g., Couch \& Sharples 1987; Bica, Alloin, \& Schmidt 1990; Charlot \& Silk 1994;LR). A valuable property of these indices particularly applicable to NGC 5018 is their virtual insensitivity to reddening. Since only neighboring spectral features are used, reddening does not affect their values 
and the embedded dust in the center of NGC 5018 will not obscure the evidence of a YSP.

In LR, the evolutionary synthesis models of Bruzual \& Charlot (1993) were used with the spectral library updated to include the higher resolution stellar library of Jacoby, Hunter, \& Christian (1984). The models were restricted to solar abundance populations only, thus metallicity effects on the indices could only be explored crudely. To remedy this, the technique now employs the evolutionary synthesis models of Worthey (1994).

The Worthey models work as follows: For a given age and metallicity, a theoretical isochrone (Bertelli et al. 1994) is consulted, with each point on the isochrone representing a parcel of stars of known luminosity, temperature, and gravity. The spectral indices for that isochrone point are interpolated from empirical fitting functions of the indices from a high-resolution spectral library (Jones 1999;see also Leitherer et al. 1996) that has been smoothed to the resolution of the NGC 5018 spectra. The indices are weighted by luminosity and number and added up along the isochrone to get the spectral index values for the entire integrated population. The population is formed from an instantaneous burst of star formation. A finite burst, however, is more realistic and will become a future feature of the models. These models were originally designed to disentangle age and metallicity effects in the integrated light of old stellar populations. To extend the age coverage to include very young ages $(<1$ Gyr) and also extend spectral coverage to the blue, the empirical library was augmented with 2103 theoretical stellar spectra computed with the Kurucz (1995) SYNTHE program. The indices were computed for each synthetic spectrum and the values were used as a lookup table with specific isochrone points being found by interpolating between the synthetic grid points (for more details see Leonardi 2000).

Figure 3 shows the Ca II index plotted against the $\mathrm{H} \delta / \lambda 4045$ index for two stellar populations with $[\mathrm{Fe} / \mathrm{H}]=-0.7$ and $[\mathrm{Fe} / \mathrm{H}]=0.0$ respectively. In the figure, the solid squares represent the index values for an instantaneous burst of star formation that has evolved to the labeled age in Gyr, so each curve follows the evolution of the indices for a stellar population of the given metallicity. Both indices initially decrease for young systems as they age, reaching a minimum at about 0.25-0.5 Gyr as the $\mathrm{O}$ and $\mathrm{B}$ stars die out and $\mathrm{A}$ stars begin to dominate the integrated light, thus generating strong Balmer lines. Subsequently, as the Balmer lines weaken, the indices increase again. Also plotted in Figure 3 are the index values observed for a select Galactic globular cluster, 47 Tuc $([\mathrm{Fe} / \mathrm{H}]=-0.7)$. The long-slit observation was acquired at the CTIO $1.5 \mathrm{~m}$ telescope in November 1995. A 10 minute exposure of 47 Tuc was acquired using the Cassegrain spectrograph with the Loral 1200x800 CCD and the B\&L grating \#58 at second order. During the exposure, the slit was trailed across the core diameter of the cluster to obtain a true integrated light measurement. The dispersion of the spectrum is $1.12 \AA /$ pixel and the resolution is FWHM $\sim 2.6 \AA$. Data reductions were done in IRAF. For details see Leonardi (2000). Although the Ca II index loses much of its age discriminating power at older ages, we still determine a reasonable globular cluster age of approximately 15 Gyr for 47 Tuc at the appropriate metallicity. Simultaneous age and metallicity discrimination with high $\mathrm{S} / \mathrm{N}$ spectra is most effective between the ages of 0.25 Gyr and 4 Gyr. Since the Ca II and $\mathrm{H} \delta / \lambda 4045$ indices are insensitive to metallicity for ages less than $\sim 0.25$ Gyr, 
the ability to uniquely determine a metallicity is lost whereas for ages greater than $\sim 4.0 \mathrm{Gyr}$, the Ca II index approaches the constant value for late type stars and its evolution essentially halts.

Unfortunately, the isochrones used here only model the horizontal branch as a red clump. To illustrate where such a population with a blue horizontal branch would fall on the Ca II- $\mathrm{H} \delta / \lambda 4045$ diagram, also included on Figure 3 are the index values for a 20 minute exposure of the Galactic globular cluster M15 taken during the same observing run as 47 Tuc and for a very metal-poor $([\mathrm{Fe} / \mathrm{H}]=-1.7)$ 15.1 Gyr model with a red clump (note that both the models in Figure 3 and the globular cluster spectra have been smoothed out to the resolution and intrinsic velocity dispersion of the NGC 5018 spectra described above). While 47 Tuc has a predominantly red horizontal branch, M15 has a blue horizontal branch (Lee 1989). The agreement between the $[\mathrm{Fe} / \mathrm{H}]=-1.7$ model and M15 is poor. Blue horizontal branch stars are luminous enough to contribute significantly the Ca II index and drive it below what the models predict for this age and metallicity.

\section{Results}

\subsection{Composite Populations}

The question we must answer for NGC 5018 is whether a YSP is contaminating the light of an old, underlying population or the light is originating from a solely old, metal-poor population. To create a composite population, we assume that the old population has an age of 15 Gyr but the metallicity can vary. We then interpolate in the index space between the old population point and a YSP point in constant increments to represent different levels of contamination by the YSP. The flux contributions for the two populations have been normalized at $4040 \AA$. The computed indices for the theoretical composite populations are illustrated in Figures 4-7. In each figure, the full evolution of the YSP is plotted in a similar manner to Figure 3, one YSP metallicity per figure. A solid, colored circle represents the index values for the 15 Gyr population denoting the old underlying stellar system, each color a different old population metallicity which may or may not be the same as the YSP's metallicity. The lines connecting the old population points with select ages along the YSP evolution curve represent composite stellar populations. The crosses along each line are interpolations between these two populations in $25 \%$ increments of the contribution of the YSP to the integrated light near $4000 \AA$. The YSP points used for interpolation were chosen so that the resulting composite population would come as closely as possible to NGC 5018.

The mean indices for the four spectra of NGC 5018 are plotted as an open triangle with error bars in Figures 4-7. Error bars for the NGC 5018 data points were calculated by computing the rms scatter among the four observations. The model trajectories were computed after both the empirical and the synthetic spectral libraries had been smoothed with gaussians to match the resolution and intrinsic velocity dispersion of the NGC 5018 spectra which allows comparison

between the theoretical integrated indices and those of NGC 5018. The observed indices for 47 Tuc and a spectrum of M32, obtained during the same observing run as the globular cluster spectra, 
are also plotted in Figures 4-7 for comparison purposes.

\subsection{Index Plots}

Figure 4 shows the index values for a very metal-poor YSP $([\mathrm{Fe} / \mathrm{H}]=-1.7)$ mixed with two possible old, underlying populations. Figure 5 does the same for a moderately metal-poor YSP $([\mathrm{Fe} / \mathrm{H}]=-0.7)$. If we also allow the old population to be metal-poor (red symbols), we have an extreme version of the case put forth by BBB of a strictly metal-poor population. If NGC 5018 were to have such a population and yet still contain the large amount of structure attributed to the galaxy by Schweizer et al. (1990), BBB postulated that NGC 5018 would either have to be the result of the merging of many metal-poor components or a large metal-poor elliptical that experienced a recent merger. The figures show quite definitively that an old stellar population with a globular cluster-like metallicity of -1.7 combined with either the very metal-poor YSP or the moderately metal-poor YSP is disallowed. Even by choosing an age of 13.2 Gyr for the "young" population to approach NGC 5018 as close as possible, the set of allowed indices defined by the possible composite populations are not near the location of NGC 5018 in the figures. No combination of metal-poor young and old populations nor a single coeval metal-poor population can reproduce the observed indices.

If a metal-poor YSP is mixed with a metal-rich old population, we have the situation depicted with the blue symbols in Figures 4 and 5. In this scenario, NGC 5018 could have evolved as a normal elliptical galaxy but then interacted with a young, metal-poor disk galaxy. Dust obscuration as suggested by Carollo \& Danziger (1994) would still be needed to explain the lack of an upturn in the UV SED. In any case, this population mixture is disallowed as well, in agreement with BBB. If a large percentage of the light is originating in the old population, the composite has a similar Ca II index value as NGC 5018 but its $\mathrm{H} \delta / \lambda 4045$ index is too weak compared to the dominant population in NGC 5018.

It is only when we allow the YSP to be metal-rich, i.e., solar or greater, that the model indices match the observed values for NGC 5018. Figure 6 shows the solar YSP curve from Figure 3 again along with four old population points, two metal-poor (green and red symbols) and two metal-rich (blue and magenta symbols). For clarity, the interpolation curves between the old population points and the YSP curve have been omitted from Figure 6 and are instead shown on Figure 7 which is identical to Figure 6 but on an expanded scale. We derive an age of $\sim 2.8$ Gyr for the YSP in NGC 5018. Also it is interesting to note in Figure 7 that the light at $4000 \AA$ is completely dominated by the YSP with virtually $100 \%$ of the light originating from the YSP regardless of the assumed old population metallicity. In fact, for an old population with $[\mathrm{Fe} / \mathrm{H}]=-1.7$, any contribution to the light by the old population will drive the model indices away from NGC 5018. Even in the most likely scenario, a metal-rich old population with a metal-rich YSP, the old population does not contribute to the integrated light. In this picture, NGC 5018 can evolve as a normal elliptical galaxy and then interact with a metal-rich companion requiring no unusual events to create the 
stellar population of NGC 5018. Thus if an underlying old metal-poor population is present in NGC 5018, it cannot be contributing significantly to the integrated blue light.

We conclude on the basis of these results that there is a young stellar population in the central regions of NGC 5018. We infer that the age of this YSP is on the order of 2.8 Gyr, the metallicity is near solar, and it is providing virtually all of the light at $4000 \AA$. These determinations are reddening independent due to the nature of the spectral indices used.

\section{Discussion}

The two observations of BBB which led them to conclude that a YSP is not present in NGC 5018 are a low $\mathrm{Mg}_{2}$ index coupled with the lack of an upturn in the UV SED both comparable to that found in M32. As seen in Figures 4-7, however, NGC 5018 lies in a different region of the $\mathrm{H} \delta / \lambda 4045-\mathrm{Ca}$ II diagram than M32 hence it cannot be just a high luminosity version of M32. With the patchiness of the dust in the central regions of NGC 5018, there may be as much as two magnitudes of extinction in the far-UV part of the spectrum which could explain the lack of an upturn (Carollo \& Danziger 1994). The results derived here, however, indicate that large amounts of dust obscuration need not be invoked, although a modest amount of extinction would bring Figure 1 into better harmony with the spectral index results. The YSP age of 2.8 Gyr derived here is reddening insensitive and thus robust regardless of the structure of the dust distribution. Also, as Figure 7 shows, the YSP is completely dominating the visible part of the spectrum of NGC 5018. A YSP of this age does not have an upturn in the UV part of the spectrum. Hence the small far-UV upturn in NGC 5018 may simply reflect the characteristics of the 2.8-Gyr-old stellar population dominating the light.

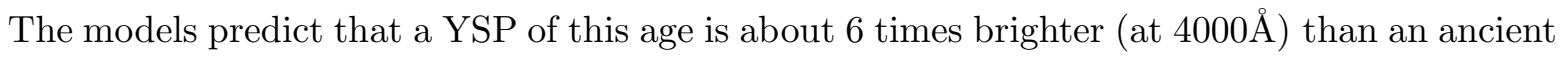
population of the same metallicity and IMF. Hence if the relative contributions from the old and young populations were only half and half, then the galaxy must be 6 parts old to 1 part young in the central region. This would qualify as a gas-rich merger event with a rapid gas inflow to the center (Weil \& Hernquist 1993) of NGC 5018. If the light contribution is weighted even more heavily toward the young population as we suggest, this implies a more violent event, perhaps a merger of two spiral galaxies or some other event involving large amounts of gas consumed in star formation, with an extremely high inflow to the nucleus. Note that, if nebular emission is filling in the Balmer lines, the derived age decreases, the YSP is brighter, and so the size of the merging event decreases.

A solar-[Fe/H] population of age 2.8 Gyr has an $\mathrm{Mg}_{2} \approx 0.20 \mathrm{mag}$, as is observed in NGC 5018 . Aging this population to 15 Gyr raises the $\mathrm{Mg}_{2}$ to $0.27 \mathrm{mag}$, still about 2 standard deviations from the mean relation for elliptical galaxies, though a dust-hidden YSP could be diluting the $\mathrm{Mg}_{2}$ index (Carollo \& Danziger 1994). We note, however, that compared to M32, NGC 5018's $\mathrm{C}_{2} 4668$ index is weaker but its $\mathrm{Mg}_{2}$ index is stronger, implying that NGC 5018 may also participate in the general 
trend for large ellipticals to have enhanced Mg abundance (e.g., Worthey 1998). This will further increase NGC 5018's $\mathrm{Mg}_{2}$ line strength as it ages so that it may one day fall among other ellipticals in the $\mathrm{Mg}_{2}-\sigma$ relation.

As mentioned previously, significant emission has been observed in the center of NGC 5018 (Goudfrooij et al. 1994). Since both of the spectral indices we used depend on the intensity in the center of a Balmer line, contamination by emission could affect the results significantly even though the contamination decreases as one moves toward higher order lines in the Balmer sequence. Specifically, emission will weaken $\mathrm{H} \delta$ relative to Fe I $\lambda 4045$ and to a lesser degree weaken Ca II $\mathrm{H}+\mathrm{H} \epsilon$ relative to $\mathrm{Ca}$ II K. Each index, if contaminated, will therefore have a higher value than if there were no emission. From Figures $4-7$, it can be seen that if emission contamination were removed, the data points for NGC 5018 would shift toward younger ages. The determined YSP age of 2.8 Gyr represents therefore an upper limit on the YSP age. The limited spectral coverage does not permit a more detailed analysis of the emission contamination, but the fact that $\mathrm{H} \beta$ from Figure 1 gives the same age indicates that the emission must be relatively modest.

The power of the method used in this paper is its ability to discriminate between different, plausible stellar populations. The verification of the presence of a YSP in NGC 5018 and its age of 2.8 Gyr are quite unambiguous, irrespective of the nature of the old, underlying population. Also, this type of determination is not unique to NGC 5018. A similar procedure can be applied to any galaxy with morphological peculiarities suspected of harboring more than one coeval stellar population. With this tool, the effects of a dynamical interaction on the integrated light of a galaxy can be analyzed more fully.

AJL would like to thank Dr. Jim Rose for a lot of guidance and many helpful discussions, Lewis Jones for providing the spectra of NGC 5018 and the referee, Dr. Scott Trager, for many helpful suggestions which improved the paper. This research was partially supported by NSF grant AST-9320723 to the University of North Carolina and by NASA through grant GO-06664.0195A awarded by the Space Telescope Science Institute which is operated by the Association of Universities for Research in Astronomy, Inc., for NASA under Contract NAS5-26555. 


\section{REFERENCES}

Bender, R., Burstein, D., \& Faber, S. M. 1993, ApJ, 411, 153

Bertelli, G., Bressan, A., Chiosi, C., Fagotto, F., \& Nasi, E., 1994, A\&AS, 106, 275

Bertola, F., Burstein, D., \& Buson, L. M., 1993, ApJ, 403, 573 (BBB)

Bica, E., Alloin, D., \& Schmidt, A., 1990, A\&A, 228, 23

Bruzual A., G., \& Charlot, S., 1993, ApJ, 405, 538

Burstein, D., Bertola, F., Buson, L. M., Faber, S. M., \& Lauer, T. R. 1988, ApJ, 328, 440

Bushouse, H. A., Lamb, S. A., \& Werner, M. W., 1988, ApJ, 335, 74

Carollo, C. M., \& Danziger, I. J., 1994, MNRAS, 270, 743

Charlot, S., \& Silk, J., 1994, ApJ, 432, 453

Couch, W. J., \& Sharples, R. M., 1987, MNRAS, 229, 423

Fort, B. P., Prieur, J.-L., Carter, D., Meatheringham, S. J., \& Vigroux, L., 1986, ApJ, 306, 110

Goudfrooij, P., Hansen, L., Jørgensen, H. E., \& Nørgaard-Nielsen, H. U., 1994, A\&AS, 105, 341

Helou, G., 1986, ApJ, 311, L33

Hernquist, L., \& Quinn, P. J., 1988, ApJ, 331, 682

Hilker, M., \& Kissler-Patig, M., 1996, A\&A, 314, 357

Jacoby, G. H., Hunter, D. A., \& Christian, C. A., 1984, ApJS, 56, 257

Jones, L. A., 1999, Ph.D. thesis Univ. of North Carolina

Jura, M., Kim, D.-W., Knapp, G. P., \& Guhathakurta, P., 1987, ApJ, 312, L11

Kim, D.-W., Guhathakurta, P., van Gorkom, J. H., Jura, M., \& Knapp, G. R., 1988, ApJ, 330, 684

Kurucz, R. L., 1995, private communication

Lee, Y.-W., 1989, Ph.D. thesis Yale University

Leitherer, C., et al., 1996, PASP, 108, 996

Leonardi, A. J., 2000, in preparation

Leonardi, A. J., \& Rose, J. A., 1996, AJ, 111, 182 (LR)

Malin, D. F., \& Carter, D., 1983, ApJ, 274, 534 
Malin, D. F., \& Hadley, B., 1997, Pub. Astro. Soc. of Aus., 14, 52

Mihos, J. C., Bothun, G. D., \& Richstone, D. O., 1993, ApJ, 418, 82

Mihos, J. C., \& Hernquist, L., 1994a, ApJ, 425, L13

Mihos, J. C., \& Hernquist, L., 1994b, ApJ, 431, L9

O'Connell, R. W., 1988, in Towards Understanding Galaxies at High Redshift, ed. R. G. Kron \& A. Renzini (Dordrecht: Kluwer), 177

Quinn, P. J., 1984, ApJ, 279, 596

Rose, J. A., 1984, AJ, 89, 1238

Rose, J. A., 1985, AJ, 90, 1927

Schweizer, F., 1983, in Proc. IAU Symp. 100, Internal Kinematics and Dynamics of Galaxies, ed. E. Athanassoula (Dordrecht: Reidel), 319

Schweizer, F., Seitzer, P., Faber, S. M., Burstein, D., Dalle Ore, C. M., \& Gonzalez, J. J., 1990, ApJ, 364, L33

Thronson, H. A., \& Bally, J., 1987, ApJ, 319, L63

Toomre, A., \& Toomre, J., 1972, ApJ, 178, 623

Trager, S. C., Worthey, G., Faber, S. M., Burstein, D., \& González, J. J. 1998, ApJS, 116, 1

Weil, M. L., \& Hernquist, L., 1993, ApJ, 405, 142

Worthey, G., 1994, ApJS, 95, 107

Worthey, G., 1998, PASP, 110, 888

Worthey, G., Dorman, B., \& Jones, L. A. 1996, AJ, 112, 948 
Fig. 1.- Two age-sensitive indices, a UV-visual color and an $\mathrm{H} \beta$ index, are plotted versus metalsensitive spectral index $\mathrm{C}_{2} 4668$. The color $\lambda 2750-V=\log F_{\lambda 2750}-\log F_{V}$ following Burstein et al. 1988 and BBB, and has units of dex rather than magnitudes. The marked dust screen extinction also refers to a straight logarithm, that is, $A_{V}=0.1 \mathrm{dex}$, or 0.25 mag. The $\lambda 2750$ passband is an average of $\mathrm{BBB}$ and Burstein et al. data from $\lambda 2650$ to $\lambda 2850$ with their extinction corrections applied. Optical spectral indices on the Lick system come from Trager et al. (1998). The optical data was taken from a significantly smaller aperture than the IUE UV data, so the $\mathrm{C}_{2} 4668$ index should be weakened somewhat (a few tenths of $\AA$ ) to compensate for this mismatch in the upper panel. Error bars in bold refer to M32 and M31 data, wider bars to the rest of the sample. Models from Worthey (1994) appear as a grid labeled by scaled solar metallicity and age in Gyr. Models are scaled solar and thus do not track element overenhancements. Such an effect probably exists for the $\mathrm{C}_{2} 4668$ index; the larger galaxies have a $\mathrm{C}_{2} 4668$ index stronger than their average abundance would dictate. In the upper panel, an arrow attached to M31 is an estimate of the effect of subtracting the hot star component (which is not included in the models) from the spectrum, as modeled by Worthey et al. (1996). The indicated effect is an underestimate because Worthey et al. maximized the contribution from very hot PAGB stars. Similar corrections should be made for NGC 3115 and NGC 3379, but we lack appropriate spectrophotometric data. NGC 3115 has a UV upturn similar to M31, but NGC 3379 has considerably more UV flux, so its correction is likely to be quite large. The other galaxies have very weak UV emission so that their corrections are negligible. Large corrections for emission fill-in in the $\mathrm{H} \beta$ index are unlikely for the galaxies plotted. If corrections are applied, galaxies move vertically to younger ages and somewhat higher abundance.

Fig. 2.- One of the long-slit spectra of NGC 5018 used to calculate the mean spectral indices. Each spectra was smoothed with a gaussian of 0.5 pixels. The absorption features used in the age-dating technique are identified.

Fig. 3.- The CaII index is plotted vs the $\mathrm{H} \delta / \lambda 4045$ index for an instantaneous burst of star formation with $[\mathrm{Fe} / \mathrm{H}]=-0.7$ (red solid and short-dashed line) and $[\mathrm{Fe} / \mathrm{H}]=0.0$ (blue long-dashed line). The colored lines represent the evolution of the index values as the population ages. Various ages (in Gyr) have been marked by solid squares. For clarity, the path from 0.004 Gyr to 0.5 Gyr on the $[\mathrm{Fe} / \mathrm{H}]=-0.7$ curve has been marked with a short-dashed line while the evolution subsequent to 0.5 Gyr has been marked with a solid line. An old, metal-poor model (green circle) is plotted to show the effects of horizontal branch morphology on the indices (see text). The error bars for the labeled globular cluster points (open triangles) are smaller than the plotting symbol. 
Fig. 4.- Ca II plotted vs $\mathrm{H} \delta / \lambda 4045$ for a composite population consisting of a metal-poor young population $([\mathrm{Fe} / \mathrm{H}]=-1.7$; evolution marked by the solid black curve, with specific ages labeled by the solid squares) and one of two OSP (age $=15.1 \mathrm{Gyr})$ populations $([\mathrm{Fe} / \mathrm{H}]=-1.7$, marked by the solid red circle and $[\mathrm{Fe} / \mathrm{H}]=0.0$, marked by the solid blue circle). Dotted lines between the old population point and the 13.2 Gyr YSP point denote interpolations between these two populations with each cross along the OSP $[\mathrm{Fe} / \mathrm{H}]=0.0$ curve marked with the fractional contribution of the YSP to the total integrated light at $4000 \AA$. The crosses along the OSP $[\mathrm{Fe} / \mathrm{H}]=-1.7$ curve are similar but have been left off for clarity. The indices for NGC 5018, M32, and 47 Tuc are plotted as open triangles.

Fig. 5.- Same as Fig. 4 but with the YSP $[\mathrm{Fe} / \mathrm{H}]=-0.7$.

Fig. 6.- Same as Fig. 4 but with the YSP $[\mathrm{Fe} / \mathrm{H}]=0.0$. Also, two other old populations have been added: $[\mathrm{Fe} / \mathrm{H}]=-0.7$ (green solid circle) and $[\mathrm{Fe} / \mathrm{H}]=+0.4$ (magenta solid circle). For clarity, the interpolations between the OSPs and the YSP have been omitted and are shown in Fig. 7 on an expanded scale.

Fig. 7.- Identical to Fig. 6 but with an expanded scale on the Ca II axis. Also, the interpolations between the OSP points and the YSP points nearest NGC 5018 are shown. The increment crosses along the $[\mathrm{Fe} / \mathrm{H}]=-0.7$ and $[\mathrm{Fe} / \mathrm{H}]=+0.0$ curves have been omitted for clarity. 


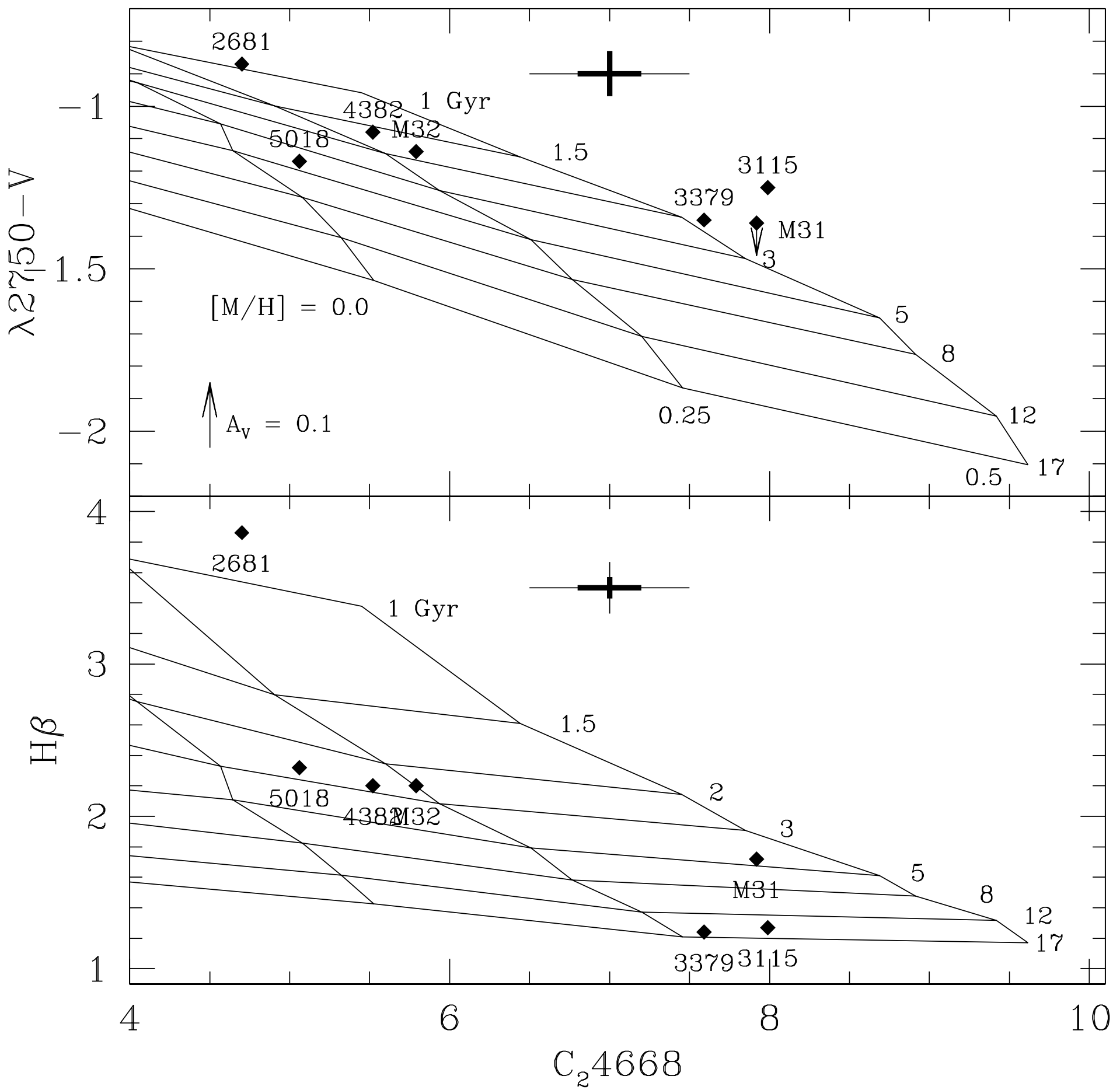




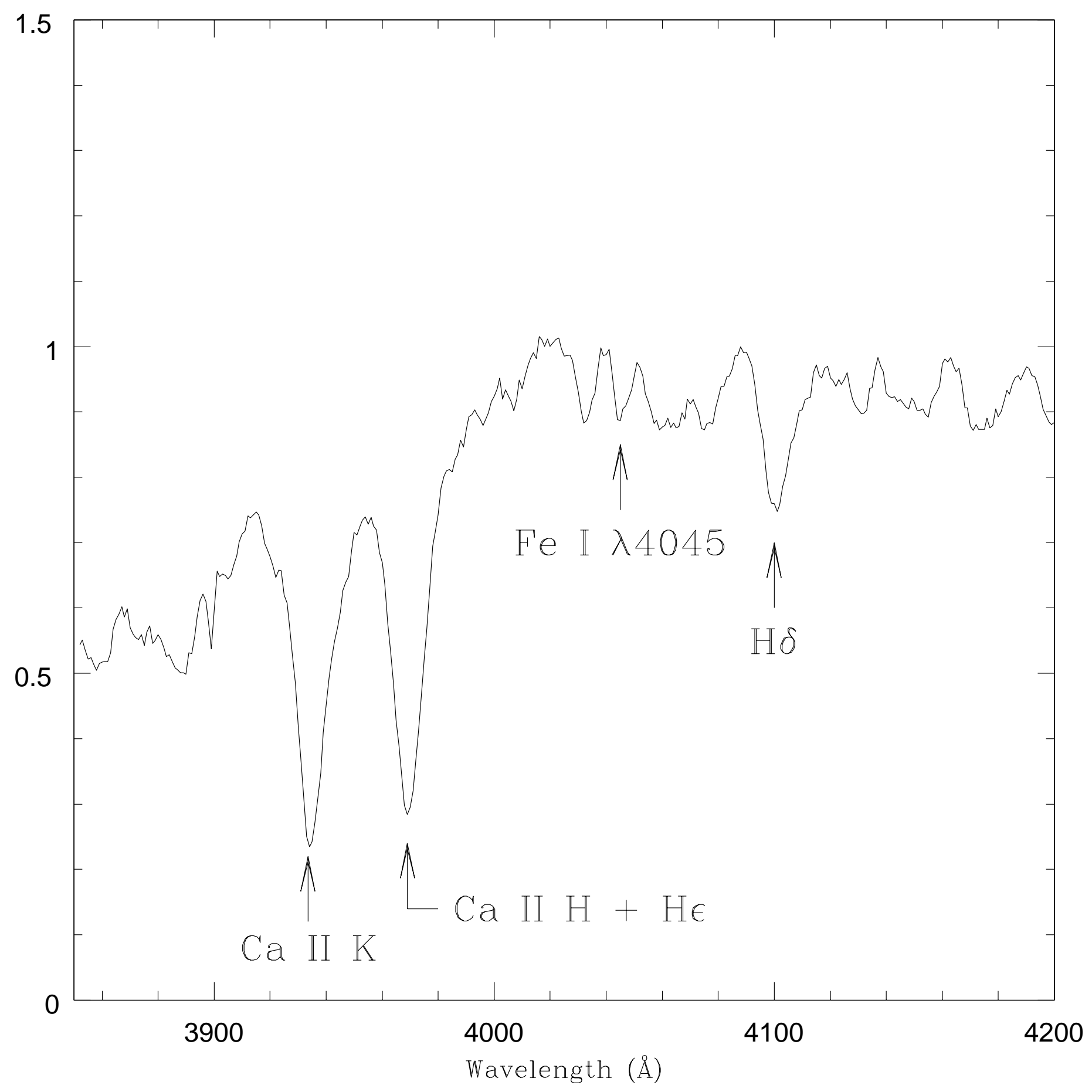




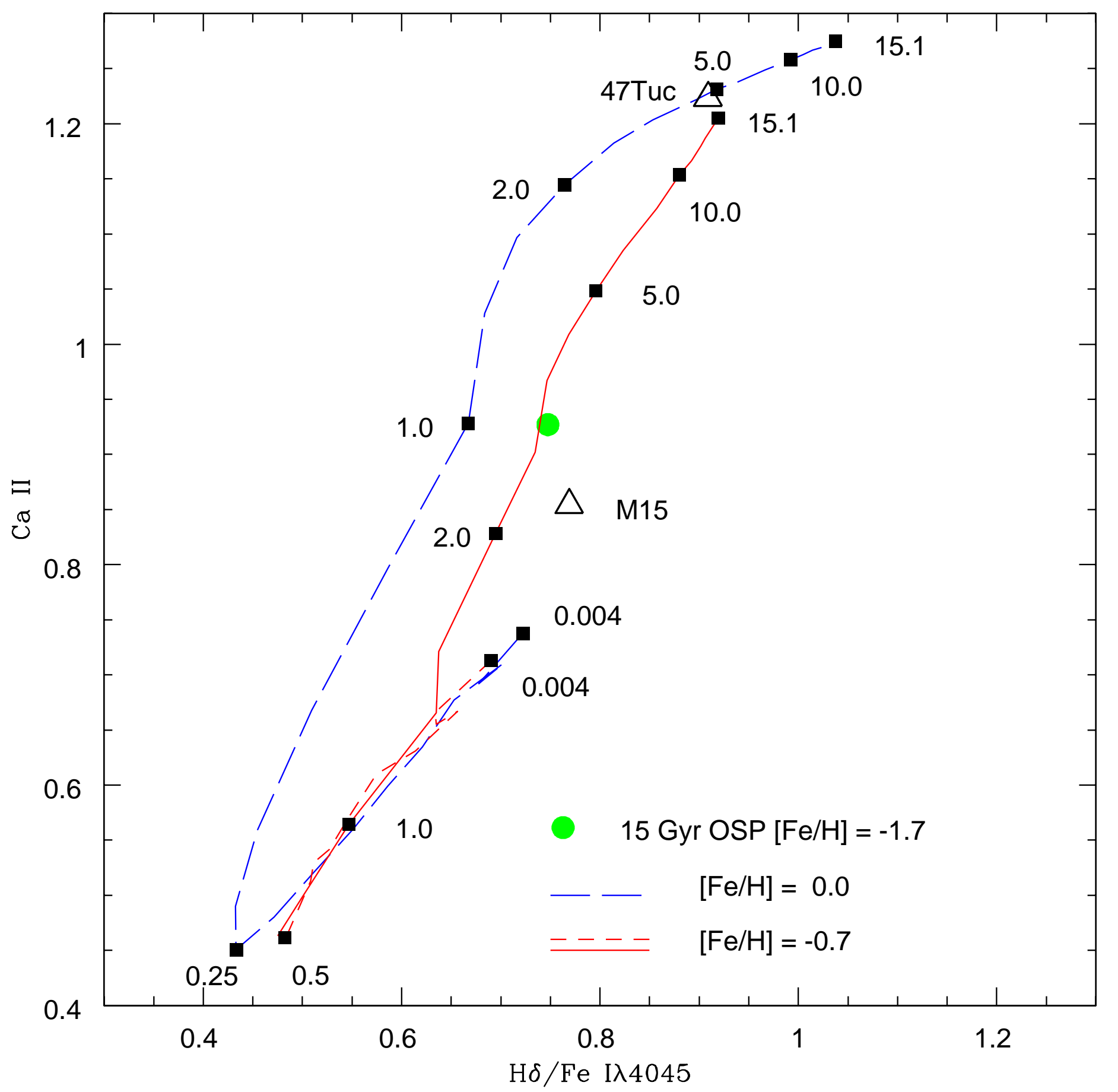




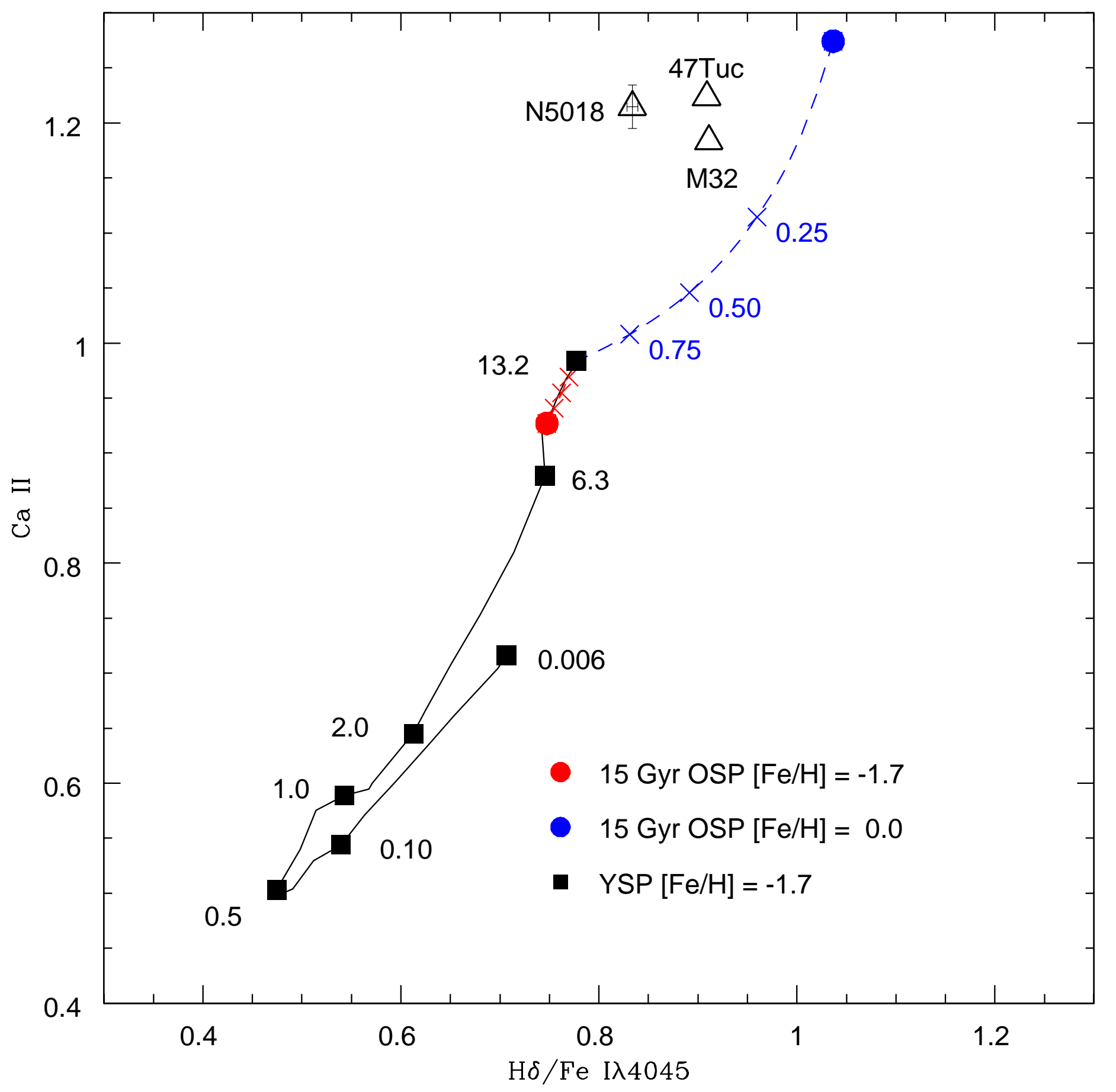




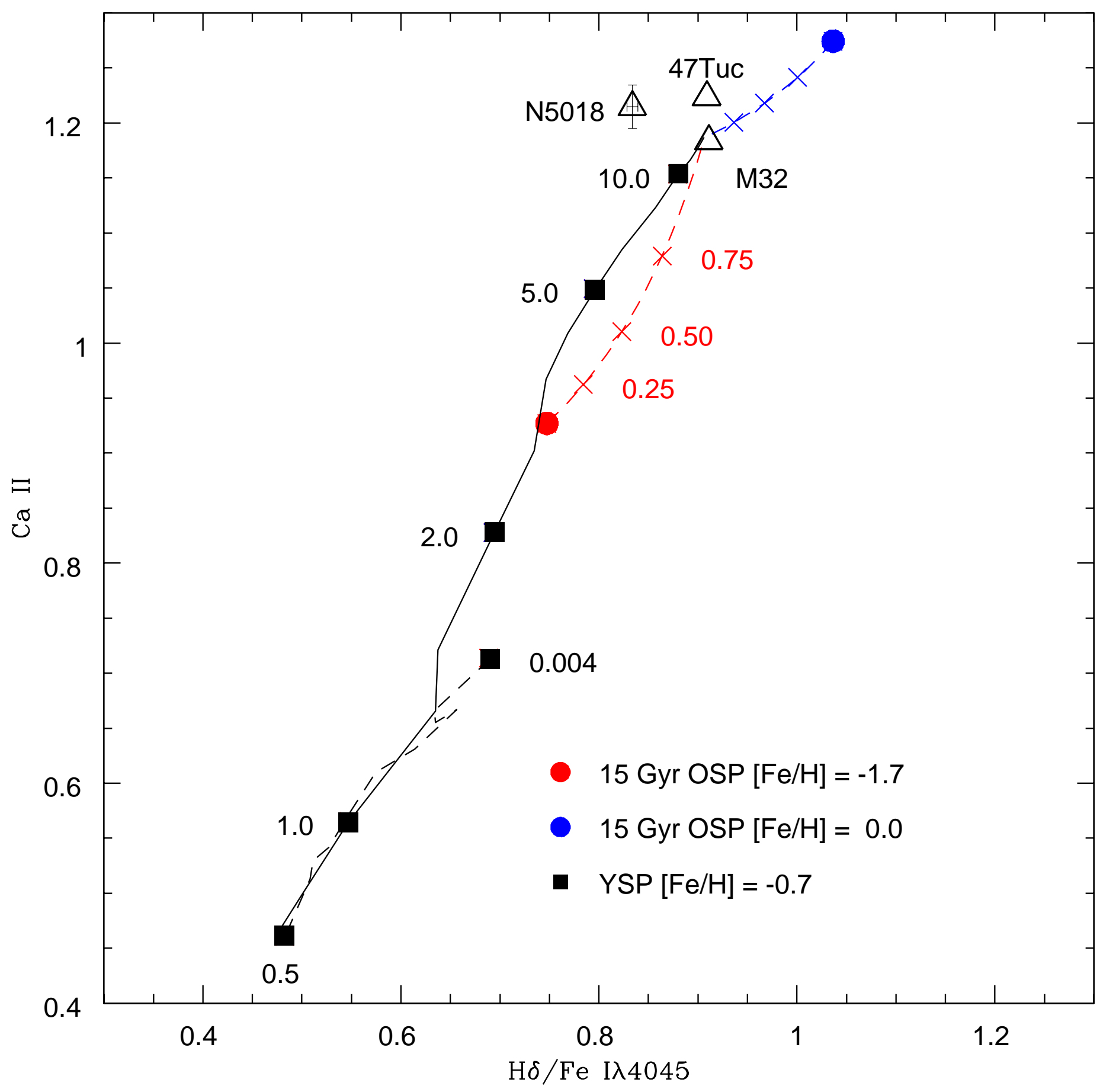




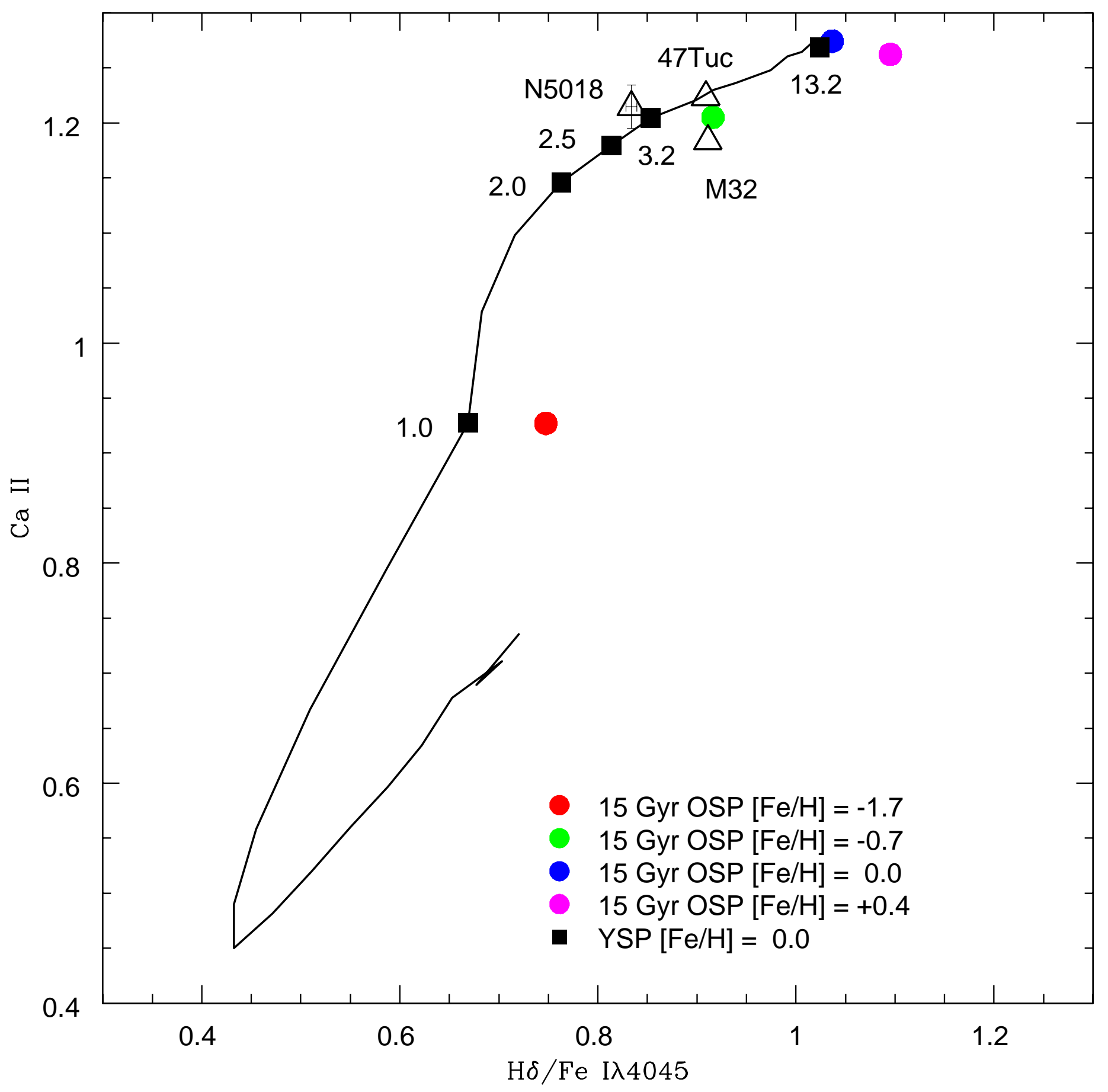




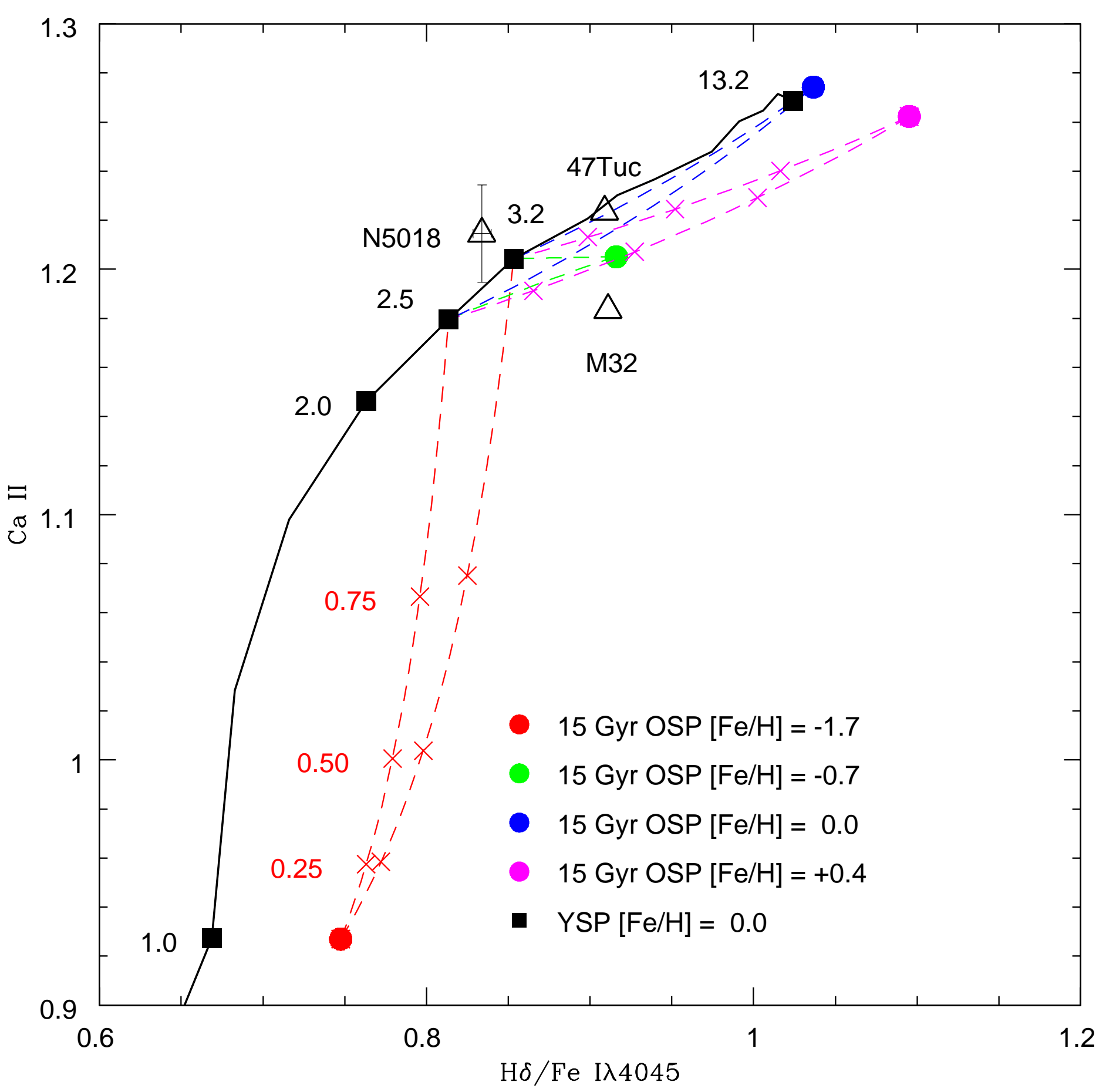

